\title{
Kinder und Jugendliche mit Asthma bronchiale im Schulsport: Teilnahmehäufigkeit abhängig vom Schweregrad oder vom asthma- spezifischen Wissen durch Schulung?
}

\author{
Influence of Asthma Severity and Asthma-Specific Knowledge on Participation in Physical Education Among \\ Children and Young People with Bronchial Asthma
}

Autoren

Institut
R. Wetzel, B. Weisser

Abteilung Sportmedizin, Christian-Albrechts-Universität zu Kiel eingereicht 17.6.2013 akzeptiert nach Revision 16.7.2013

\section{Bibliografie}

Dol http://dx.doi.org/ 10.1055/s-0033-1344596

Online-Publikation: 2.9.2013

Pneumologie 2013; 67: 567-572

(c) Georg Thieme Verlag KG

Stuttgart · New York

ISSN 0934-8387

\section{Korrespondenzadresse Prof. Dr. med. Burkhard Weisser}

Christian-Albrechts-Universität zu Kiel

Institut für Sportwissenschaft Abt. Sportmedizin

Olshausenstraße 74

24098 Kiel

bweisser@email.uni-kiel.de

\section{Zusammenfassung \\ $\nabla$}

Hintergrund: Sport und Bewegung sind eine wichtige Säule der Asthmatherapie. Auch wenn bei fast allen Asthmatikern im Kindes- und Jugendalter durch körperliche Belastung eine Bronchokonstriktion ausgelöst werden kann, ist die möglichst vollständige Teilnahme am Schulsportunterricht das Ziel. In der vorliegenden Untersuchung soll geklärt werden, wie regelmäßig die Teilnahme am Schulsport ist. Weiterhin sollte der Einfluss von Asthmaschweregrad sowie des asthmaspezifischen Wissens untersucht werden. Methode und Patienten: Es wurden 217 Schülerinnen und Schüler der Jahrgangsstufen 5-10 mit Asthma mittels eines Fragebogens befragt. Erhoben wurden u.a. die Teilnahmehäufigkeit am Schulsportunterricht, das asthmaspezifische Wissen, Teilnahme an einer Schulung sowie belastungsspezifische subjektive Symptome im Schulsportunterricht.

Ergebnisse: Eine regelmäßige Teilnahme am Schulsport konnte bei rund $80 \%$ der Befragten festgestellt werden. Ein Zusammenhang zwischen Asthma-Schweregrad und Teilnahme am Schulsport bestand nicht. Es ergab sich hingegen eine Korrelation der Teilnahmehäufigkeit $(r=$ $0,35, \mathrm{p}<0,01)$ mit dem asthmaspezifischen Wissen. Das asthmaspezifische Wissen war bei den Befragten mit einer Asthmaschulung (19\% der Gesamtgruppe) signifikant besser. Das Asthmawissen muss insgesamt aber als schlecht bezeichnet werden. Nur 58 Schüler kannten die Lippenbremse, 112 nahmen trotz vergessenem Asthmaspray am Sportunterricht teil.

Schlussfolgerung: Die vorliegende Untersuchung zeigt, dass die Teilnahme am Schulsport mit dem asthmaspezifischen Wissen korreliert, nicht jedoch mit dem Asthma-Schweregrad. Unsere Da-

\section{Abstract \\ $\nabla$}

Background: Physical activity is an important part of the therapy of bronchial asthma. Even if there is exercise-induced bronchoconstriction in more or less all children with bronchial asthma, the goal is an almost complete participation in physical education lessons in school. The present study investigates their participation rate. In addition, the influence of the knowledge about asthma and the severity of the disease were studied.

Methods: A questionnaire on asthma knowledge and severity, participation rate and exercise-induced symptoms was used in 217 school children suffering from asthma (9-16 years).

Results: $80 \%$ of the patients showed a regular participation in physical education. No correlation between participation and asthma severity was found. In contrast, a significant correlation between participation and knowledge about asthma could be demonstrated $(\mathrm{r}=0.35, \mathrm{p}<0.01)$. The knowledge about asthma was better in school children who had completed any asthma education program in the past (19\%). However, there was generally an insufficient knowledge about asthma and exercise in the investigated group. 58 subjects were not familiar with pursed lip breathing and 112 participated in physical education lessons in spite of forgotten asthma medication.

Conclusion: Our study demonstrates a moderate increase of participation in physical education lessons in school children with bronchial asthma during the last ten years. The participation rate correlated with asthma-specific knowledge, but not with asthma severity underlining the importance of education programs on asthma and exercise. 
ten ergeben eine leichte Zunahme der regelmäßigen Teilnahme am Schulsport in den letzten 10 Jahren und demonstrieren diesbezüglich einen Effekt der Schulung zum Thema Sport und körperliche Belastung bei Asthma bronchiale.

\section{Einleitung}

In Deutschland stellt Asthma bronchiale eine der häufigsten chronischen Erkrankungen im Kindesalter dar, bis etwa 10\% der Kinder und Jugendlichen sind betroffen [1-3]. Die deutsche Atemwegsliga nennt aktuell eine Zahl von 2-5 asthmakranken Kindern pro Schulklasse [4]. Die in den letzten 30 Jahren gestiegene Prävalenz der Atemwegserkrankung hat im Schulalltag vor allem Auswirkungen auf den Sportunterricht, da fast alle Asthmatiker im Kindes- und Jugendalter ein Anstrengungsasthma haben. Beim Anstrengungsasthma löst körperliche Belastung die Bronchokonstriktion aus und stellt somit die für den Schulsport wichtigste Form des Asthmas dar.

Gerade vor dem Hintergrund der sinkenden sportlichen Aktivitäten in der Freizeitgestaltung von Kindern und Jugendlichen gewinnt der Schulsport zunehmend an Bedeutung [1]. Dies gilt nicht nur hinsichtlich der Verbesserung der körperlichen Leistungsfähigkeit, sondern ebenso in Bezug auf die psychische und soziale Entwicklung. Die Verbesserung der Fitness kann mit einer Reduktion von Asthmaanfällen mittels Modifikation der Anfallsschwelle einhergehen. Eine möglichst umfangreiche Teilnahme aller Schülerinnen und Schüler am Schulsport und somit auch und gerade jener, die an Asthma bronchiale leiden, sollte oberstes Ziel sein.

Dennoch zeigt ein Blick auf die Teilnahmezahlen, dass viele Asthmatiker immer noch vom Sportunterricht dauerbefreit sind, nur eingeschränkt teilnehmen oder hohe Fehlzeiten aufweisen [5]. Ein Grund hierfür könnte eine „overprotection“ durch das Elternhaus darstellen. Andererseits konnten wir und andere auch mangelnde Kenntnisse von Sportlehrkräften im Zusammenhang mit Asthma und Sport zeigen [6,7]. Genauso jedoch spielt ein nicht ausreichendes Wissen bei den betroffenen Kindern und Jugendlichen selbst in Bezug auf ihre Erkrankung eine entscheidende Rolle. Denn hieraus resultiert oft ein inadäquater Umgang mit der Atemwegserkrankung in Form von falscher Schonung oder gefährlicher Überforderung.

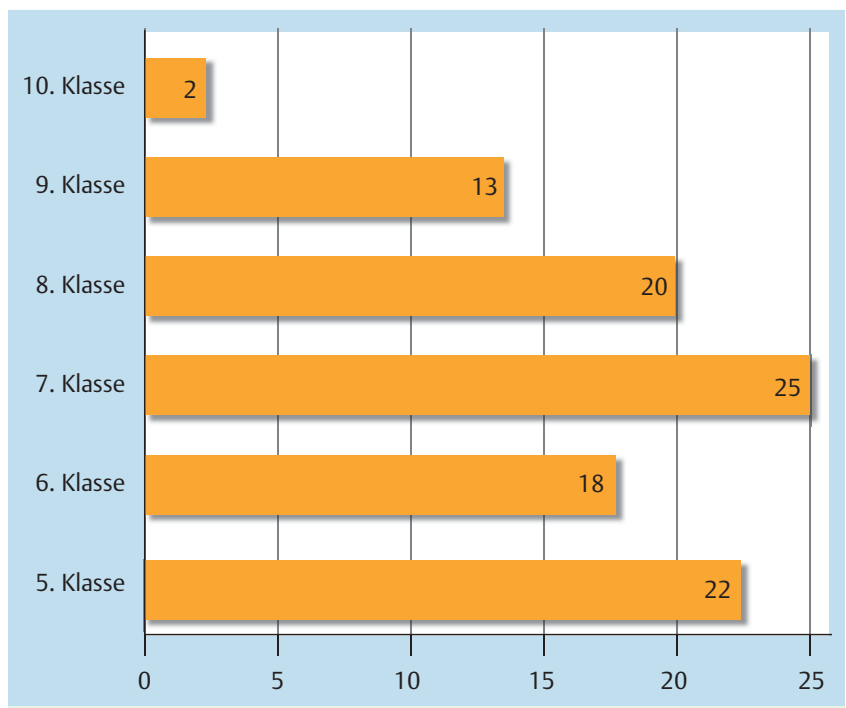

Abb.1 Verteilung der Teilnehmer auf die Klassenstufen 5-10 in Prozent.

Ziel der vorliegenden Studie war zunächst die Erhebung der aktuellen Teilnahmehäufigkeit am Schulsport in einer Gruppe von 217 Schülerinnen und Schülern mit Asthma bronchiale ( $\bullet$ Abb.1). Weiterhin sollte untersucht werden, ob eher der Asthmaschweregrad oder das asthmaspezifische Wissen und die Teilnahme an Schulungsprogrammen mit der Teilnahmehäufigkeit am Schulsport korrelieren.

\section{Material und Methode \\ $\nabla$}

Grundlage der Untersuchung ist eine von Mai bis Juli 2011 mittels standardisiertem Fragebogen durchgeführte Befragung von 217 Schülerinnen und Schülern mit Asthma bronchiale der Klassenstufe 5-10 ( $\bullet$ Abb.2) an Gemeinschaftsschulen und Gymnasien in Schleswig-Holstein sowie vereinzelt in Niedersachsen und Nordrhein-Westfalen. Die Auswahl der teilnehmenden Schulen, in denen die Befragung asthmatischer Schüler stattfand, erfolgte zufällig. Ebenso erfolgte die Auswahl der teilnehmenden

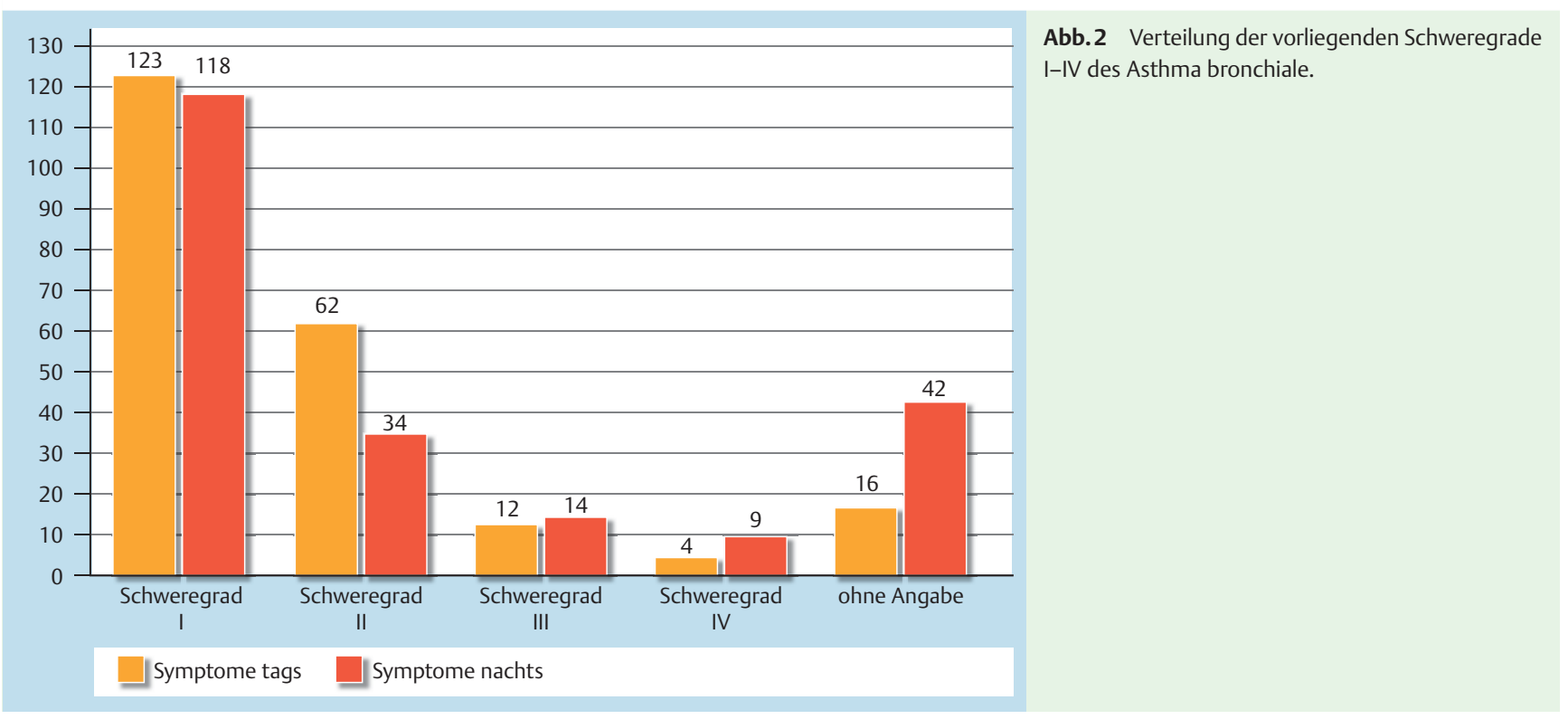


Klassen in Abhängigkeit von Verfügbarkeit und Integrierbarkeit der Befragung in den Unterrichtsverlauf. Eine erste Kontaktaufnahme wurde telefonisch oder per Email über das Schulsekretariat durchgeführt, mit der Bitte um Weiterleitung an Schulleitung und Fachbereichsleitung Sport, bzw. in einem nächsten Schritt an das gesamte Sportkollegium. Die Grundlage der Auswahl der zu befragenden Schüler war die Selbstangabe über ihre Erkrankung. Die Auswahl der Klassenstufen 5-10 ist einerseits durch die Verfügbarkeit andererseits auch durch das Manifestationsalter von Asthma bronchiale begründet. Der verwendete Fragebogen ist vollstandardisiert, und die Antworten sind nach dem Prinzip des multiple choice fest vorgegeben. Es werden zwei Typen von Fragekategorien verwendet: Im ersten Fall ergibt sich ein Ausschluss der einzelnen Antwortkategorien untereinander, im zweiten Fall sind Mehrfachnennungen möglich. Die befragten Schüler wurden hierauf vor der Befragung hingewiesen. Die insgesamt 18 Fragen gliedern sich in 7 thematische Sektoren. Sektor 1 erfragt allgemeine Hintergrundinformationen des Schülers (Alter, Geschlecht, Schulform, erfolgte Schulungen und Rehabilitationsmaßnahmen zu Asthma bronchiale). Sektor 2 beinhaltet die Anamnese des Asthma bronchiale (Schweregrad, Form). Sektor 3 ermittelt die Selbsteinschätzung der Schüler bezüglich ihres asthmaspezifischen Wissensstandes und prüft das tatsächliche Wissen über Definitionen des Asthmas, Vorboten und anfallsauslösende Stimuli und Notfallverhalten im Anfall. Sektor 4 erfasst die Relevanz von Sport für Asthmatiker, Sektor 5 das Belastungsempfinden im Sportunterricht. Im Sektor 6 sind Art und Umfang der Teilnahme am Sportunterricht von Interesse, während im Sektor 7 geeignete Belastungsformen für Asthmatiker sowie praktische Aspekte des Sportunterrichts erfasst werden.

Die Ermittlung des asthmaspezifischen Wissens erfolgte durch eine Übersetzung von Antworten aus den Sektoren 3, 4 und 7 in Punktwerte, die eine Gesamtpunktzahl für richtig angekreuzte Antworten ergab und folglich eine Vergleichbarkeit der Befragten untereinander erlaubte. Die Klassifizierung des Asthma-Schweregrads erfolgte nach den Angaben in Sektor 2 des Fragebogens und den Kriterien der Deutschen Atemwegsliga [4].

\section{Ergebnisse}

Im Rahmen der Untersuchung wurden 217 Schüler an insgesamt 36 Schulen (28 in Schleswig-Holstein, 6 in Niedersachsen, 2 in Nordrhein-Westfalen) befragt, die nach Selbstauskunft an Asthma bronchiale litten oder aufgrund der Symptomlage als begründete Verdachtsfälle galten. Von den 217 Befragten waren 117 männlich und 92 weiblich bei einem Durchschnittsalter von 12,9 Jahren. Die Differenz der Summe zu 217 ist durch nicht gemachte Angaben seitens der Befragten zu erklären. Dieses gilt ebenso für alle Folgeergebnisse. Bei etwa gleicher Verteilung der befragten einzelnen Klassen pro Jahrgangsstufe liegen wesentlich mehr Ergebnisse in den Klassen 5-8 vor, sodass die geringere Prävalenz der Erkrankung mit zunehmender Annäherung an das Erwachsenenalter eine Rolle spielen könnte.

Von den 217 Befragten gaben nur 41 (19\%) an, bereits an einer Schulung oder Rehamaßnahme zu Asthma bronchiale teilgenommen zu haben. Die Annahme, dass diejenigen, die bereits an einer Schulung u.ä. teilgenommen hatten, über mehr und bessere Kenntnisse hinsichtlich ihrer eigenen Erkrankung verfügten, konnte durch inferenzstatistische Verfahren bestätigt werden. So erzielte diese Gruppe im Durchschnitt 11,2 Punkte im Wissenstest und lag hiermit 3,3 Punkte über dem Ergebnis, das jene im Mittel erzielten, die noch an keiner asthmaspezifischen Schulung teilgenommen hatten $(\mathrm{p}<0,05)$.

Ein umfangreiches asthmaspezifisches Wissen ist bei Kindern und Jugendlichen für einen richtigen Umgang mit ihrer Erkrankung unerlässlich. Dies gilt in besonderem Maße für körperliche Aktivität und somit für den Schulsport, da die belastungsinduzierte Bronchokonstriktion im Kindes- und Jugendalter eine hohe Prävalenz aufweist. Kenntnisse hinsichtlich der richtigen Anwendung des Sprays, richtiger Belastungsformen sowie entsprechend gestalteter Aufwärmphasen erhöhen die Teilnahme am Sportunterricht und gestalten diese effizienter. Deshalb wurde untersucht, ob Asthmatiker mit einem umfangreicheren Wissen auch eine höhere Teilnahme am Schulsport aufwiesen.

Es ergab sich ein signifikanter Unterschied bezüglich der Teilnahme von Asthmatikern im Sportunterricht dahingehend, dass eine höhere Punktzahl im asthmaspezifischen Wissenstest auch eine regelmäßigere Teilnahme implizierte. Schüler, die das Item „Ich nehme immer teil“ ankreuzten, wiesen bessere Kenntnisse bezüglich individueller Trigger, geeigneter Belastungsformen, des idealen Stundenaufbaus, der Bedeutung des Aufwärmens sowie des richtigen Verhaltens bei Atemnot und Anfall nach. Ebenso wussten sie besser Bescheid bei der täglichen Feststellung der aktuellen Lungenleistungsfähigkeit und dem richtigen Umgang mit der Medikation.

Der Schweregrad des Asthmas der befragten Schüler war deshalb von Interesse, da untersucht wurde, ob sich das Belastungsempfinden im Sportunterricht in Abhängigkeit von der Häufigkeit der Symptome gegenüber nicht-asthmatischen Mitschülern unterscheidet und ob schwergradige Asthmatiker während der körperlichen Belastung mehr Angst haben, einen Asthmaanfall zu erleiden.

Es konnte bestätigt werden, dass Asthmatiker, die einen Schweregrad > I hatten, dreimal so oft angaben, sich im Sportunterricht aufgrund der aufkommenden Atemnot unwohl zu fühlen und einen Asthmaanfall zu fürchten.

Das ermittelte tatsächliche asthmaspezifische Wissen der Schüler ergab, dass gerade einmal $2 \%$ über ihre eigene Erkrankung gut informiert waren, 44\% mittelmäßig und das Wissen von mehr als der Hälfte der Befragten als schlecht einzuordnen war $(\bullet$ Abb.3).

Besondere Relevanz erhalten diese Ergebnisse durch den Vergleich mit der Selbsteinschätzung. 31\% hielten sich für gut informiert, 55\% für mittelmäßig und gerade einmal 14\% erachteten

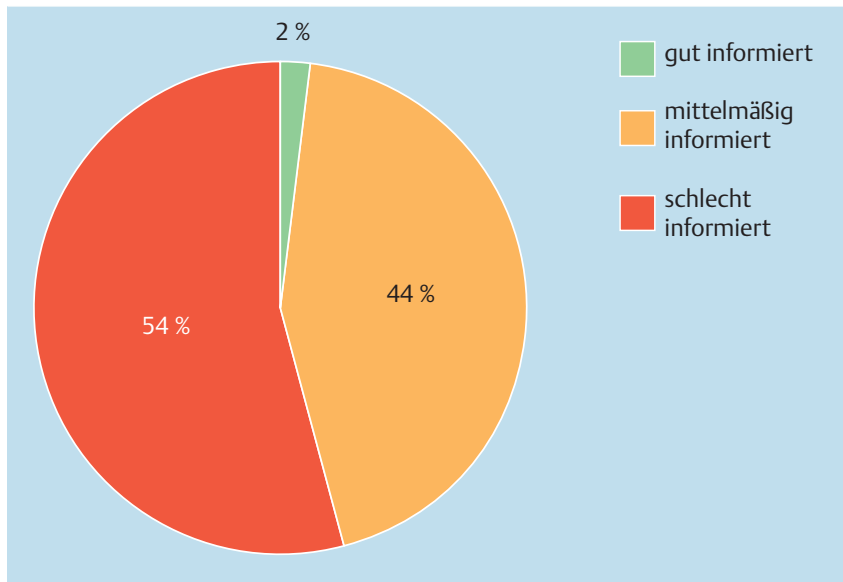

Abb.3 Ergebnis des Wissenstests in Prozent. 


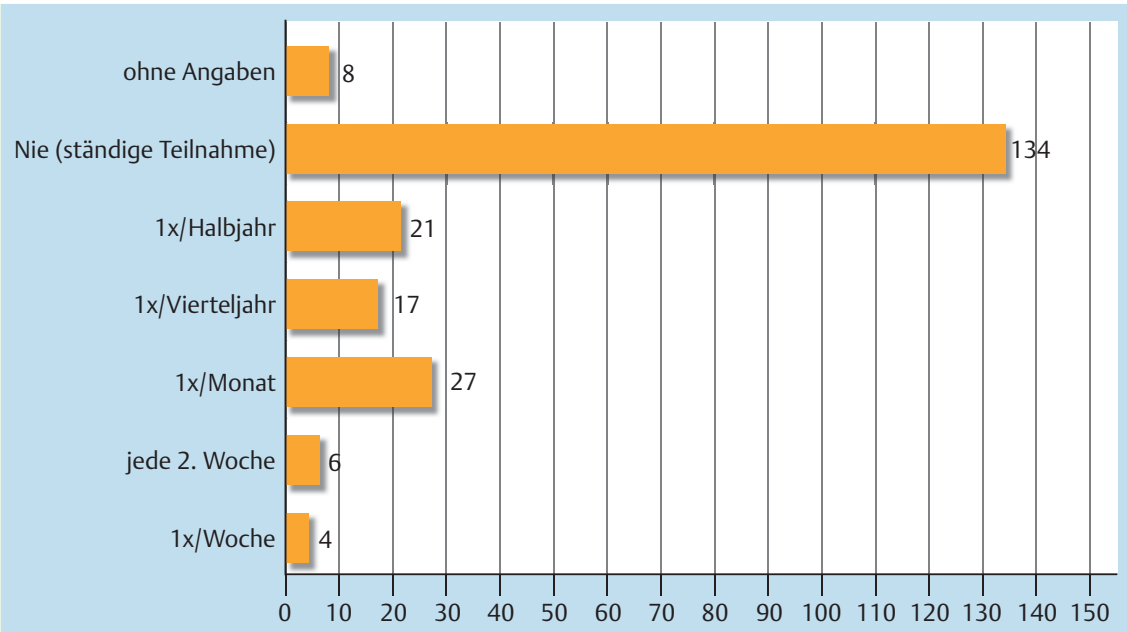

Abb.4 „Wie oft nimmst du durchschnittlich NICHT am Sportunterricht teil?"

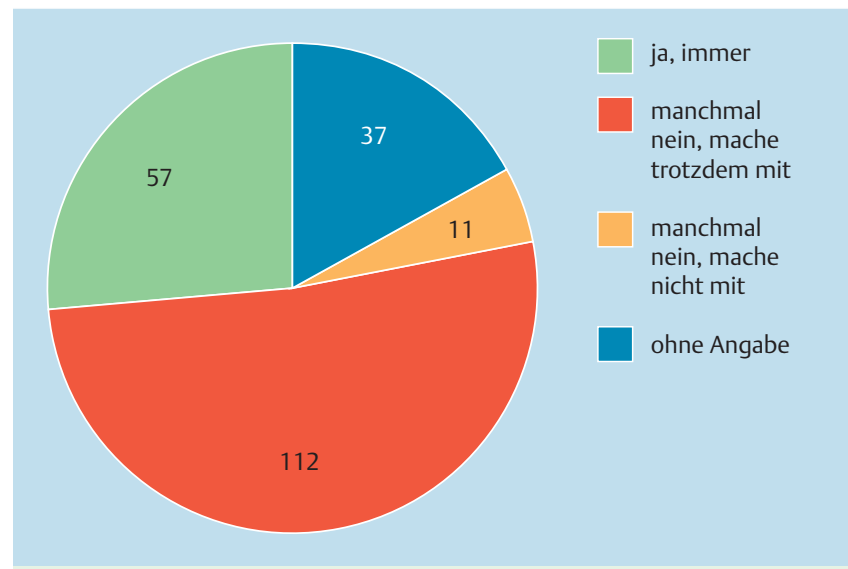

Abb.5 Thema Asthmaspray: „Hast du deins immer dabei?“

ihr asthmaspezifisches Wissen als schlecht. Somit war sich der Großteil der Befragten seiner Defizite nicht bewusst ( $\bullet$ Tab. 1). So wussten gerade einmal 39 (18\%) von 217 Befragten, dass physikalische Auslöser wie Kälte oder Nebel als Trigger für ein hyperreagibles Bronchialsystem fungieren können. 199 (92\%) der befragten Schüler wurden so ermittelt, die nicht wussten, welche klimatischen Bedingungen für sie als Asthmatiker während einer körperlichen Aktivität geeignet sind und welches die zu meidenden Witterungsverhältnisse sind. Der Asthmaanfall und in der Folge zu ergreifende Maßnahmen waren den Schülern ebenfalls nicht ausreichend bekannt. Nur 58 (27\%) Asthmatiker wussten um den Mechanismus der Lippenbremse, nur 71 (33\%) kannten atemerleichternde Stellungen wie den Kutschersitz oder die Torwarthaltung.

Weiter war von Interesse, wie viele der Befragten Anfallsanzeichen richtig wahrnehmen und deuten können. In Bezug auf die körperliche Belastung während einer Sportstunde hielten 70 (32\%) Schüler das Gefühl des „Außeratemseins“ beim erwärmenden Hopserlauf für Anfallsvorboten. Wie bei Williams et al. [10] wurde bestätigt, dass viele asthmatische Schüler anstrengungsbedingte Reaktionen ihres Körpers fehlinterpretierten und eine belastungsbedingte Mehratmung ihrem Asthma zuschreiben.

Für den Asthmatiker geht es vor allem darum, durch regelmäßiges Sporttreiben die Schwelle, an der asthmatische Symptome auftreten, möglichst anzuheben und eine größere Toleranz der bronchialen Schleimhaut gegenüber anstrengungsbedingter
Tab. 1 Selbsteinschätzung im Verhältnis zur tatsächlichen erzielten Wissenspunktzahl.

\begin{tabular}{|llll|}
\hline Selbsteinschätzung & \multicolumn{3}{l|}{ erzielte Wissenspunkte } \\
\hline & $0-8$ & $9-17$ & $18-25$ \\
\hline gut informiert & 25 & 37 & 4 \\
\hline mittelmäßig informiert & 71 & 49 & 1 \\
\hline schlecht informiert & 21 & 9 & 0 \\
\hline Gesamt & 117 & 95 & 5 \\
\hline
\end{tabular}

Mehratmung und dem daran gekoppelten Kältereiz herbeizuführen. Dennoch meinte ein Viertel der Befragten, von Sport sei bei Anstrengungsasthma abzusehen. Sport zur Stärkung der Atemwegsmuskulatur hielten nur 64 (30\%) für sinnvoll.

Ferner konnte die tatsächliche Teilnahme und entsprechende Fehlzeit von Asthmatikern im Sportunterricht ermittelt werden. Hierbei gaben $79 \%$ der befragten Schüler asthmabedingte Fehlzeiten an, die zweimal im Schulhalbjahr nicht übertrafen $(\bullet$ Abb.4).

Neben dem Umfang der Teilnahme wurde ebenso ihre Art erhoben. Belastungen mit Intervallcharakter haben gegenüber obstruktionshervorrufenden Dauerbelastungen für Asthmatiker den Vorteil der optimalen Ausnutzung der Refraktärphase, die nach anfänglicher belastungsbedingter Hyperventilation auftritt. Dieses zeigte sich deutlich in den Zahlen, da nur 3\% Abbrüche bei Laufspielen und Sprints auf eine gute Toleranz von Intervallbelastungen schließen lassen. Dem gegenüber standen 16\%, die angaben, bei längeren Laufbelastungen am Stück abbrechen zu müssen.

Das Asthmaspray sollte ein ständiger Begleiter eines jeden Asthmatikers bei körperlicher Aktivität im Alltag sein. Nur 57 (26\%) Schüler gaben jedoch an, ihr Spray immer dabei zu haben. Wird das Spray vergessen, so stellt sich die eigenverantwortliche Frage, ob man trotzdem teilnimmt. 112 (52\%) Schüler bejahten dies. Nur 11 (5\%) gaben an, bei vergessenem Spray auch nicht teilzunehmen $(\bullet$ Abb.5).

Zusammenfassend zeigte der Großteil der befragten Asthmatiker ein unzureichendes Wissen bezüglich ihrer eigenen Erkrankung. Die Teilnahme am Sportunterricht war eher vom asthamaspezifischen Wissen als vom Schweregrad abhängig. Schülerinnen und Schüler nach einer Schulung wiesen ein besseres Wissen auf. 


\section{Diskussion}

\section{V}

Asthma bronchiale verhinderte bei 79\% der Betroffenen die Teilnahme am Sportunterricht nur bis zu zweimal im Schuljahr, was als regelmäßige Teilnahme definiert wurde. Es verblieben somit immerhin $21 \%$, die regelmäßig wöchentlich oder monatlich beschwerdebedingt fehlten, obwohl durch eine medikamentöse Therapie ein fast vollständiger Anfallsschutz erreicht werden könnte [8,9]. Bei Meyer et al. nahmen 2001 60\% der Asthmatiker uneingeschränkt am Sportunterricht teil [5], sodass unsere Daten nach 10 Jahren einen gewissen Fortschritt dokumentieren.

Williams et al. stellten 2008 die zunehmende körperliche Inaktivität von Asthmatikern in Folge falscher Vorstellungen hinsichtlich des Umganges mit ihrer Erkrankung fest [10,11]. Sie wiesen auf die resultierenden Defizite im koordinativen und konditionellen Grundlagenbereich hin. Entsprechende Mängel waren mehr als zwei Dritteln unserer Befragten nicht klar. Konsequenzen sind häufig eine Verschlechterung des Krankheitsbildes in Folge ihrer geringen Fitness, soziale Ausgrenzungsvorgänge und Minderwertigkeitskomplexe bei den Betroffenen.

In nationalen und internationalen Therapierichtlinien wird deshalb die „normale Ausübung des Schulsports“ als ein Ziel der Asthmatherapie angegeben [8]. Wo liegen nun die Gründe dafür, dass die Befragung keine regelmäßige Teilnahme von 100\% ergab? Zur Beantwortung wurde der Zusammenhang zwischen asthmaspezifischem Wissenstand und Teilnahme untersucht.

Das Ergebnis des Wissenstests, besonders der Fragen nach Verhaltensmaßnahmen im Notfall, ist bedenklich. Lippenbremse oder die atemerleichternde Haltung Kutschersitz waren im Mittel gerade einmal 20\% der befragten Schüler bekannt. Eine deutlich nachlassende Wirkung des Asthmasprays deuteten nur $10 \%$ als Vorbote eines Asthmaanfalls. Nur unwesentlich mehr Schülern war klar, dass ein Abfall des Peak-Flows von mehr als 20\% eine alarmierende Verschlechterung der Lungenfunktion darstellt.

In unserer Untersuchung differierten Selbsteinschätzung und Realität bezüglich Asthmawissen erheblich. Von 217 Schülern schätzten lediglich 74 (34\%) ihren Kenntnisstand richtig ein. Trotz Korrelation von Wissenspunktzahl und Schulsportteilnahme ergab sich jedoch insgesamt auch noch eine recht hohe Teilnahme am Sportunterricht bei extrem niedriger durchschnittlicher Wissenspunktzahl. 112 Schüler (52\%) gaben z.B. an, trotz vergessenem Asthmaspray Sport zu treiben, und waren sich über die Bedeutung des Sprays im Notfall nur selten bewusst. Die Konsequenzen für die Sportpraxis waren aus dem ermittelten Belastungsempfinden abzulesen. So gaben mehr als die Hälfte der Asthmatiker an, dass ihr Belastungsempfinden und das Befinden während der Sportstunde vorwiegend von Angst und Panik vor Anfällen dominiert wurden. Trotz der Annahme, dass schwergradige Asthmatiker signifikant häufiger von asthmatischen Beschwerden betroffen sein müssten, konnte dies nur für das Item „Angst vor einem Asthmaanfall“ bestätigt werden. Ein Engegefühl in der Brust sowie Panik vor der Atemnot beschrieben insgesamt 80 Schüler (36\%). Hierunter waren jedoch nicht signifikant mehr Schüler mit einem Schweregrad größer 1.

Es nahmen zwar 172 (79\%) Schüler regelmäßig am Sportunterricht teil, dies jedoch mit deutlichen Beschwerden, was nicht zuletzt am unzureichenden asthmaspezifischen Wissen lag. Diese Daten unterstützen die Forderung nach Teilnahme aller Asthmatiker an Asthmaschulungen. Unterstützt wird dies durch die besseren asthmaspezifischen Kenntnisse der bereits geschulten Befragten. Diese nahmen zwar nicht signifikant häufiger am Sport- unterricht teil, taten dies jedoch mit deutlich weniger Beschwerden sowie weniger Angst- und Panikgefühlen. Eine Teilnahme im vollen Umfang ohne Fehlzeiten kann also nur aus einem guten asthmaspezifischen Kenntnisstand erwachsen.

Williams et al. führten außerdem den geringen Wissensstand der Lehrkräfte als Grund dafür an, dass die Sportstunden in nur 4,7\% der Fälle „einen spezifischen Stundenaufbau [zeigten], der es den Asthmatikern erlaubt, ohne Einschränkung der Atmung teilzunehmen“ [10,11]. Diese Ergebnisse decken sich mit unserer Untersuchung: In 79 Fällen (36\%) war der Sportlehrer nicht über die Atemwegserkrankung informiert, und 161 Asthmatiker/-innen $(74 \%)$ verneinten die regelmäßige Kontrolle des Notfallsprays durch den Lehrer. Gerade wenn die Sportlehrer nicht ausreichend informiert sind, müssen die Betroffenen es selber sein und können auf erste Anzeichen von Atemnot durch Anwendung der Lippenbremse u.ä. richtig reagieren. Ein gutes Wissen stärkt das Selbstbewusstsein und reduziert Ängste. Voraussetzung ist jedoch, dass Belastungsempfinden und Körperwahrnehmung durch vielfältige Bewegungserfahrungen geschult werden. Dann kann auf Anfallsvorboten mit Maßnahmen wie Lippenbremse oder atemerleichternden Haltungen reagiert werden.

Unbestritten ist, dass die körperliche Aktivität von Asthmatikern unzureichend ist und dass ein regelmäßiges Training auch wegen der hohen Co-Prävalenz von Adipositas ein Eckpfeiler der Therapie sein könnte [12-15].

Bei der Interpretation unserer Daten müssen einige Limitationen beachtet werden. Die Auswahl der Teilnehmer mit Asthma erfolgte aufgrund von Selbstangaben. Eine Diagnosesicherung durch den behandelnden Arzt konnte jedoch im Rahmen dieser Untersuchung nicht erfolgen. Auch die Einteilung in Schweregrade kann kritisch hinterfragt werden, zumal Fehlklassifikationen nicht vollständig ausgeschlossen werden können. Eine andere Einteilung nach Kontrollgrad wäre möglicherweise relevanter für Lebensqualität und Leistungsfähigkeit [16]. Ferner führte in unserer Untersuchung ein besseres asthmaspezifisches Wissen zu häufigerer Teilnahme am Sportunterricht ebenso wie die geschulten Asthmatiker auch besser über ihre Erkrankung informiert waren. Trotzdem war die Teilnahmehäufigkeit der geschulten Kinder und Jugendlichen nicht signifikant unterschiedlich. Dies kann jedoch auch statistische Gründe haben, da nur ein vergleichsweise geringer Anteil eine Schulung durchlaufen hatte (19\%). Weitere Untersuchungen müssen klären, welche Faktoren neben dem Schulungsgrad für eine geringere Sportteilnahme (z.B. Migrationshintergrund, sozioökonomischer Status) verantwortlich sein könnten.

Die Ergebnisse können unter Berücksichtigung der genannten Einschränkungen wie folgt zusammengefasst werden: Rund $80 \%$ der Asthmatiker nehmen überwiegend regelmäßig am Sportunterricht teil. Die hohen Teilnahmezahlen scheinen auf den ersten Blick einen Fortschritt gegenüber früheren Untersuchungen darzustellen [5]. Sie verbergen jedoch ein leichtsinniges Verhalten, denn die Teilnahme am Schulsport erfolgte trotz erheblicher Defizite im asthmaspezifischen Wissen der Erkrankten. Die Schüler mit Asthma gingen hohe Risiken ein, belasteten sich nicht angemessen und hätten ihr Spray im Bedarfsfall häufig nicht dabei. Die Konsequenzen waren regelmäßige asthmatische Beschwerden und in der Folge Ängste vor einem Anfall.

Unsere Daten unterstützen die Forderung nach regelmäßigen Asthmaschulungen für Asthmatiker aller Schweregrade. Darüber hinaus sollten Bewegungsschulungen zur Verbesserung der Körperwahrnehmung stattfinden. Geschulte Asthmatiker zeigten bessere Kenntnisse und konnten mit weniger Beschwerden und 
Ängsten Sport treiben. Für eine regelmäßige und verantwortungsbewusste Teilnahme am Sportunterricht von Schülerinnen und Schülern mit Asthma bronchiale ist somit eher ein differenziertes asthmaspezifisches Wissen als der Schweregrad der Erkrankung entscheidend.

\section{Interessenkonflikt}

Die Autoren geben an, dass kein Interessenkonflikt besteht.

\section{Ergänzendes Material}

Den Fragebogen Asthma bronchiale im Schulsport finden Sie im Internet unter http://dx.doi.org/10.1055/s-0033-1344596.

\section{Literatur}

1 Lechler J. Trägt das veränderte Bewegungsverhalten von Kindern und Jugendlichen zur Entstehung chronischer Krankheiten bei? Deutsche Zeitschrift für Sportmedizin 2008; 59: 241 - 242

2 Jaeschke $R$. Effekte von körperlichem Training bei Kindern und Jugendlichen mit Formen des Asthma bronchiale und/oder der Adipositas: Meta-Analysen empirischer Studien von 1950 bis 2002. Band 1-3. Deutsche Sporthochschule Köln/Institut für Kreislaufforschung und Sportmedizin; 2006

3 Berzel HG. Asthma bronchiale bei Kindern und Jugendlichen: Basiswissen, Hintergrundinformationen, Krankheitsbilder erkennen und erfolgreich therapieren. Stuttgart: Wiss. Verl.-Ges; 2003

4 Deutsche Atemwegsliga e.V. In der deutschen Gesellschaft für Pneumologie. http://www.atemwegsliga.de/asthma.html
5 Meyer A, Machnick MA, Behnke W et al. Teilnahme von asthmakranken Kindern am Schulsport: Eine Erhebung an Hamburger Schulen. Pneumologie 2002; 56: 486-492

6 Forck I, Märzhäuser A, Weisser B. Asthma bronchiale und Sport. Wissensstand der sportunterrichtenden Grundschullehrer/innen in Schleswig-Holstein über Asthma bronchiale. Pneumologie 2008; 62: $226-230$

7 Sandsund M, Thomassen M, Reinertsen RE et al. Exercise-induced asthma in adolescents: challenges for physical education teachers. Chron Respir Dis 2011; 8: 171 - 179

8 Bacharier LB, Boner A, Carlsen K et al. Diagnosis and treatment of asthma in childhood: a PRACTALL consensus report. Allergy 2008; 63: 5 34

9 Lecheler J, Biberger A, Pfannebecker B. Asthma \& Sport: Theoretische Grundlagen und praktische Handlungsanleitungen. 13th ed. Berchtesgaden: INA-Verl; 2006

10 Williams B, Hoskins G, Pow J et al. Low exercise among children with asthma: a culture of over protection? A qualitative study of experiences and beliefs. British Journal of General Practice 2010; 60: 319 326

11 Williams B, Powell A, Hoskins $G$ et al. Exploring and explaining low participation in physical activity among children and young people with asthma: a review. BMC Family Practice 2008; 9: 40

12 Dogra S, Baker J, Ardern CI. The role of physical activity and body mass index in the health care use of adults with asthma. Annals of Allergy, Asthma \& Immunology 2009; 102: $462-468$

13 Glazebrook C, McPherson AC, MacDonald IA et al. Asthma as a barrier to children's physical activity: implications for body mass index and mental health. Pediatrics 2006; 118: 2443 - 2449

14 Lang DM, Butz AM, Duggan AK et al. Physical activity in urban schoolaged children with asthma. Pediatrics 2004; 113: e341-6

15 Taylor B, Mannino D, Brown C et al. Body mass index and asthma severity in the National Asthma Survey. Thorax 2008; 63: 14-20

16 Humbert M, Holgate S, Boulet $L$ et al. Asthma control or severity: that is the question. Allergy 2007; 62: 95-101 